\title{
Poesía catalana digital: la edición crítica electrónica de la obra de Francesc Fontanella ${ }^{1}$
}

\author{
Marc Sogues \\ Pep Valsalobre \\ Universitat de Girona. Institut de Llengua i Cultura Catalanes \\ marc.sogues@udg.edu \\ pep.valsalobre@udg.edu
}

Recepción: 11/05/2017, Aceptación: 06/06/2017, Publicación: 22/12/2017

\begin{abstract}
Resumen
En los últimos años, dentro del ámbito catalán, la crítica textual está avanzando considerablemente y buena parte de estos avances se están produciendo en el medio digital. El presente artículo ofrece un repaso de los proyectos de edición crítica que se están desarrollando actualmente y se centra en el primero que ha sido publicado en internet: la edición crítica de la poesía completa de Francesc Fontanella, realizada por miembros de Nise, el grupo de investigación en literatura catalana de la Edad Moderna de la Universitat de Girona. En el presente artículo se detallan las prestaciones que ya ofrece la edición y se apuntan tanto sus limitaciones como sus expectativas de desarrollo en el futuro.
\end{abstract}

\section{Palabras clave}

Ecdótica; edición crítica; edición filológica; crítica textual; filología catalana; Francesc Fontanella; poesía catalana del barroco

\begin{abstract}
Catalan digital poetry: The electronic critical edition of the work of Francesc Fontanella In recent years, textual criticism has progressed considerably within the Catalan context, and much of these advances are occurring in digital environments. This article presents a review of the projects of critical edition that are currently being developed and focuses on the first of these projects that has been published on the Internet: the critical edition of the complete poetry of Francesc Fontanella carried out by members of Nise, the research group of the Universitat de Girona in Catalan literature of Modern Era. This
\end{abstract}

1. Este trabajo se inscribe en el proyecto de investigación del Ministerio de Economía y Competitividad, ref. FFI2015-70095-P. Para más detalles sobre este proyecto, véase Valsalobre (2015a). 
article details the benefits of the edition in its current state, and points out both its limitations and its future expectations for development.

\section{Keywords}

Ecdotics; critical edition; philological edition; textual criticism; Catalan philology; Francesc Fontanella; Catalan Baroque poetry

La creación de los entornos digitales ha abierto para la filología en general, y para la crítica textual en particular, un universo de nuevas posibilidades. En ellos, la intertextualidad se ha convertido en hipertextualidad, o lo que es lo mismo, ha dejado de ser una propiedad del texto para pasar a ser una propiedad estructural del canal a través del cual este llega al lector. Esto ha generado una nueva gramática, la del hipertexto, que permite que el texto literario pueda ser fácilmente amplificado, complementado o expandido mediante su enlace con contenidos de todo tipo y formato.

En las grandes literaturas del planeta existen, desde hace ańos, proyectos que utilizan los recursos digitales para crear aproximaciones innovadoras a aspectos como la génesis, la transmisión o la fijación de los textos de sus autores canónicos, así como para generar herramientas y prestaciones web que facilitan al investigador la compilación sistemática de datos de interés filológico. El abanico de propuestas, en este sentido, es muy amplio y las características de los proyectos, muy distintas, de manera que no existe un modelo único, sino más bien iniciativas que organizan sus diseños y sus contenidos de formas diversas en función de los objetivos que persiguen. Así, tan posible es encontrar proyectos de digitalización exhaustiva de obras completas, traducciones, manuscritos y todo tipo de documentación relacionada con el autor, como en el caso de The Walt Whitman Archive, ${ }^{2}$ como hallar proyec-

2. Véase: <http://whitmanarchive.org>. 
tos — con un enfoque determinado (edición genética)— dedicados específicamente a una obra concreta (Madame Bovary), ${ }^{3}$ bibliotecas digitales que ofrecen versiones anotadas del texto en formato llano, como la del proyecto TC/12, ${ }^{4}$ dedicada al teatro clásico español, o ediciones críticas anotadas en formato hipertextual y que permiten la consulta de los testimonios digitalizados, como es el caso de la edición de La dama boba realizada por Prolope. ${ }^{5}$

Sea como fuere, lo que es seguro es que los proyectos que menos abundan en la red son los de las características de este último caso, es decir, las ediciones críticas que presentan textos publicados directamente en el medio digital, sin pasar antes por el formato papel, que aprovechan las oportunidades del hipertexto y que ofrecen el lector la posibilidad de acceder a los datos referentes a la fijación crítica del texto. Nos referimos, en fin, a aquellos trabajos más directamente inspirados por los modelos tradicionales de la crítica textual. Esta situación es hasta cierto punto comprensible, por un lado, porque es lógico (y positivo) que se utilice el nuevo medio para construir formas de acercamiento al texto distintas de las que ofrece el papel; por otro lado, también es verdad que los grandes autores de las principales literaturas del mundo suelen tener ya un corpus bien fijado y establecido, por lo que ya no resulta tan urgente resolver sus problemas ecdóticos.

En cambio, en literaturas de dimensiones más modestas, como la catalana, todavía quedan muchos autores y obras que esperan ser presentados en ediciones fiables. Sin duda, en buena medida, esta circunstancia, sumada a la reducción de costes y a las mayores expectativas de difusión que ofrece el medio digital, explica por qué las principales iniciativas de carácter filológico que se están gestando actualmente en el seno de la academia catalana se orientan hacia la publicación digital de ediciones críticas de los principales autores de los distintos periodos históricos.

El objeto del presente artículo es, precisamente, dar a conocer una de estas iniciativas: la edición crítica electrónica de la obra completa del poeta y dramaturgo barcelonés Francesc Fontanella (1622-1682/83). A tales efectos, en primer lugar, nos referiremos brevemente a los principales proyectos existentes en la internet literaria catalana que, como el nuestro, tienen una clara vocación filológica y surgen del ámbito académico. A continuación, explicaremos las motivaciones y los objetivos de nuestra edición, y describiremos las prestaciones y funcionalidades que ofrece la actualidad. Para terminar, señalaremos las limitaciones que detectamos en el trabajo realizado en las primeras fases del proyecto, y apuntaremos las que creemos que son sus posibles expectativas de mejora en el futuro.

3. Véase: <http://www.bovary.fr>.

4. Véase: $<$ http://tc12.uv.es/>.

5. Véase: <http://damaboba.unibo.it>. 


\section{Las ediciones críticas electrónicas en la literatura catalana}

Hasta la década de 1980, la crítica textual dentro del ámbito catalán tuvo un desarrollo bastante desigual, a causa de las circunstancias histórico-políticas que dificultaron la implantación de los estudios sobre la lengua y la literatura propias en el ámbito académico. Sin embargo, desde entonces hasta ahora, la disciplina ha experimentado un salto cualitativo notable, hasta el punto de que, dentro de sus siempre modestas posibilidades, podemos decir que en estos primeros compases del nuevo siglo pasa por un buen momento, en el que coexisten distintas generaciones de editores filológicos bien preparados y en activo, y se publican con normalidad tanto ediciones críticas de autores clásicos como manuales sobre edición crítica de reconocida solvencia. ${ }^{6}$

Dentro de la academia catalana, existen tres grandes grupos de investigación cuyos trabajos se orientan preferentemente hacia la edición filológica de textos en formato digital — Narpan, Nise y la Cátedra Màrius Torres_ que cubren todo el arco temporal de la historia literaria, puesto que se dedican, respectivamente, al estudio de la época medieval, moderna y contemporánea. En los últimos quince años, estos grupos han llevado a cabo varias iniciativas para trasladar a la red la experiencia editorial acumulada. En el caso de Nise, esta experiencia ha cristalizado ya en la publicación de la edición que aquí presentamos, mientras que, en los otros dos casos, como veremos a continuación, los resultados están previstos en un futuro próximo.

Narpan es un grupo interuniversitario formado por investigadores de la Universitat de Barcelona, la Universitat Autònoma de Barcelona y la Universitat de Girona. ${ }^{7}$ Fueron pioneros en el salto al medio digital ya que, en el año 2000, en colaboración con investigadores de la Università di Napoli Federico II, crearon el Repertorio informatizzato dell'antica letteratura catalana (RIALC), ${ }^{8}$ un inventario crítico de la poesía catalana de los siglos XIV y XV. La mayor parte de los textos y de los aparatos de variantes de este repertorio proceden de ediciones publicadas anteriormente en papel, cuya referencia se especifica al final de cada texto, aunque, en algunos casos - los menos-, textos o aparato fueron mejorados o corregidos, o incluso preparados expresamente para su publicación web.

6. Véanse, por ejemplo, manuales como los de Martínez-Gil (coord.) (2013) y Veny-Mesquida (2015).

7. Véase <http://www.narpan.net>.

8. Véase en $<$ http://www.rialc.unina.it>. 


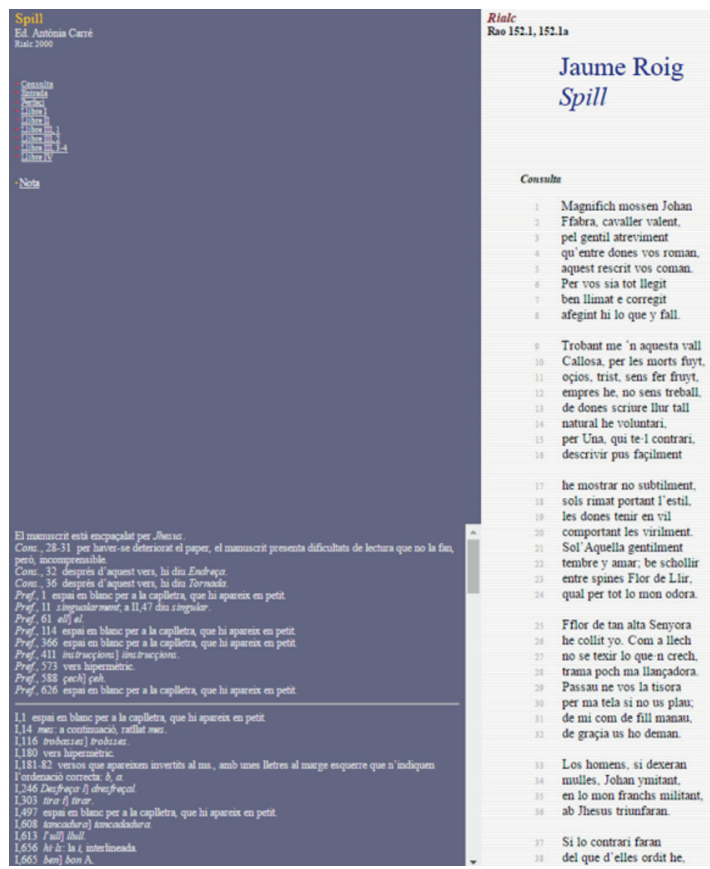

Figura 1.

Ejemplo de un fragmento de la novela satírica Espill, (c. 1460) de Jaume Roig, con el correspondiente aparato crítico en el lateral de la página. Como puede observarse en la parte superior izquierda, en este caso, el aparato, de Antònia Carré, fue preparado expresamente para su publicación en el RIALC, en el año 2000.

A pesar del interés de sus contenidos, de su rigor filológico y del volumen del corpus publicado - RIALC sigue siendo la mayor biblioteca de textos literarios de época medieval en lengua catalana que podemos encontrar hoy en día en internet-, el Repertorio presenta textos en formato llano y ofrece al lector unas posibilidades limitadas de interacción para con el texto. Estas limitaciones se deben, en parte, a que se trata de una web de cierta antigüedad, pero, sobre todo, al hecho de que Narpan no ha continuado desarrollando este espacio porque, en los últimos tiempos, el equipo ha dedicado sus esfuerzos a un proyecto más ambicioso: la creación de una biblioteca digital propia, en formato xtf, que permitirá la publicación de textos en formato xml, editados a través de TEI (Martí 2014).9 En esta plataforma, el grupo tiene previsto poder ofrecer, en los

9. <http://www.narpan.net/bibliotecadigital/biblio-digital.html>. 
próximos ańos, un amplio corpus de textos medievales de edición propia, con aparato de variantes, notas críticas y motores de búsqueda, y está previsto que, a largo plazo, acabe incorporando también todo el corpus procedente del RIALC, que adoptará estas mismas características.

También en fase de desarrollo se encuentra el segundo de los grandes proyectos de edición crítica electrónica existentes en el ámbito catalán: Ėcdosi, impulsado por Joan Ramon Veny-Mesquida desde la Cátedra Màrius Torres de la Universitat de Lleida. Se trata de lo que podríamos denominar un editor automático en línea que, partiendo de la digitalización exhaustiva de todos los testimonios disponibles y de su transcripción digital vía OCR — pero revisada y corregida-, permitirá al usuario generar una edición crítica dinámica, es decir, una versión del texto en la cual el lector podrá escoger como base cualquiera de los testimonios y verá construirse el aparato de variantes — de tradición, genético, evolutivo- en relación a este. El usuario registrado, además, podrá personalizar su escritorio seleccionando las ventanas que quiere que se le muestren (texto/imagen del testimonio, aparatos críticos y de notas, foro de debate) y estableciendo el tipo y el nivel de anotación que desea tener a la vista, lo cual amplía el abanico de usuarios no solo al lector erudito o al investigador sino a todo aquel que busca textos fiables. El aplicativo, además, facilitará las búsquedas y la consulta de concordancias, así como el acceso a la consulta de diccionarios en línea para los resultados de las búsquedas. Este proyecto, que ha supuesto ya la digitalización prácticamente completa de ediciones y manuscritos de autores de los siglos XIX y xx como Oller, Salvat-Papasseit, Rosselló-Pòrcel, Torres, Riba, Espriu, Garcés y Manent, entre otros, se encuentra en una fase avanzada y, aunque por ahora todavía no se encuentra en acceso abierto, está previsto que próximamente lo esté. ${ }^{10}$

A la espera, pues, del desarrollo de la biblioteca digital de Narpan y de $\grave{E} c$ dosi, la edición crítica electrónica de Fontanella que Nise está llevando a cabo actualmente en la Universidad de Girona, bajo la coordinación de los autores del presente artículo, ha de ser considerada la primera de ámbito catalán que aparece en la red. Veamos, a continuación, con más detalle, las circunstancias que han motivado su realización y las posibilidades que ofrece al lector.

\section{Nise y la edición crítica de textos de época moderna}

Nise es un grupo interdisciplinario formado por investigadores de distintas universidades catalanas, españolas e internacionales, que se consolidó en la Universitat de Girona durante la década de 1990 bajo el impulso y el maestrazgo del catedrático Albert Rossich. En aquel momento, la literatura catalana de los siglos

10. El acceso a Ėcdosi se podrá realizar a través de la web de la Càtedra Màrius Torres (<http:// www.catedramariustorres.udl.cat>). 
modernos seguía estigmatizada por el discurso decadentista; ${ }^{11}$ muchas de las obras de la época continuaban inéditas o solo estaban disponibles en ediciones de escasa fiabilidad. En parte, esto explica por qué, desde el principio, la crítica textual fue uno de los ejes centrales de la actividad del grupo, ya que, al fin y al cabo, sin una versión filológicamente fiable, difícilmente se puede llegar a emitir un juicio crítico justo sobre un texto. Ahora bien, a pesar de esta orientación preferente hacia la ecdótica, la de Nise ha sido siempre una aproximación holística, que aspira a combinar los enfoques de todas las ramas de la filología, esto es, además de la ecdótica, la historia literaria, la teoría literaria y la literatura comparada. En esta línea, cabe mencionar, además de la recuperación de la obra poética de Vicent Garcia (1985; Rossich 1985), el estudio y edición de autores como Agustí Eura (2002), Joan Ramis (2002) o Pau Puig (2012), o la recuperación de la literatura femenina de los siglos XVI-XVIII (Zaragoza 2016), así como la antología Poesia catalana del barroc (Valsalobre y Rossich 2006), que ofrecía por primera vez una edición a la vez rigurosa y divulgativa de poesía en catalán del periodo barroco.

Desde sus inicios, uno de los principales objetivos de Nise ha sido divulgar los resultados de sus investigaciones, puesto que el grupo ha sido siempre consciente del gran desconocimiento que, tanto dentro del mundo académico como entre el gran público, existía de la literatura producida en las tierras de lengua catalana durante los siglos del Renacimiento, del Barroco y de la Ilustración. Esta voluntad divulgativa se ha concretado en la elaboración de distintos manuales dedicados a la historia literaria del periodo, ${ }^{12}$ pero también, y de forma creciente, en la actividad del grupo en el medio digital. Esta actividad empezó en el año 2009 con la creación de la página del grupo (<www.nise.cat>), que ponía a disposición del usuario tres herramientas fundamentales: la base de datos «Poesia en cançoners», ${ }^{13}$ el repertorio bibliográfico Qüern, de ediciones y estudios sobre la lengua y la literatura catalana medieval y moderna, ${ }^{14}$ y la biblioteca digital, que incluía textos en formato llano editados por miembros del grupo, además

11. Sobre la génesis y los efectos del discurso decadentista, véanse Rossich (1997) y Rossich y Valsalobre (2011: 11-19).

12. En este sentido, hay que destacar publicaciones panorámicas como Literatura i cultura catalanes (segles XVI-XVIII) (Rossich y Valsalobre 2007), el tercer volumen del Panorama critic de la literatura catalana (Rossich 2011), la miscelánea de estudios Del Cinccents al Setcents. Tres-cents anys de literatura catalana (Miralles 2010) o Literatura catalana moderna (siglos XVI-XVIII) (Rossich y Valsalobre 2011).

13. Véase <http://www.nise.cat/ca-es/ca-es/bdpoesiaencançoners/sobreaquestabasededades.aspx>. Esta base fue el principal resultado del proyecto «Inventario general de la poesía catalana del barroco en cancioneros manuscritos (ss. XVII-XVIII)» (MEC. Dirección General de Investigación - HUM2006-08326/FILO). Recoge la información mínima imprescindible para la fácil localización de las composiciones poéticas dispersas en manuscritos con tal de ofrecerlas a la comunidad científica y así establecer las bases de proyectos futuros de edición de textos poéticos y otras investigaciones críticas.

14. Véase $<$ http://www.quern.cat $>$. 
de facilitar el acceso a otros textos catalanes del periodo moderno presentes en internet. La página fue ampliando sus contenidos y, en el año 2013, obtuvo el reconocimiento público al serle concedido la concesión exaequo del Premi Lletra a la mejor iniciativa digital sobre literatura catalana "por su papel a la hora de dar relevancia histórica y literaria a un periodo de nuestra literatura generalmente difamado, como es el que incluye el Renacimiento, el Barroco y la Ilustración». ${ }^{15}$

Al mismo tiempo que daba el paso al mundo digital, el grupo empezó a orientar su atención hacia el que, juntamente con Vicent Garcia, es, a todas luces, el autor más completo y de mayor ambición literaria de las letras catalanas de la época moderna: el poeta y dramaturgo barcelonés Francesc Fontanella (1622-1682/83). Con la voluntad de ahondar en el estudio de la vida y la obra de Fontanella, en 2006 Nise organizó en la Universitat de Girona un congreso monográfico dedicado al autor, en el cual participaron filólogos, historiadores, historiadores del arte e historiadores de la literatura, cuyo resultado más material fue un volumen misceláneo de estudios (Valsalobre y Sansano 2006). El éxito de aquella jornada desveló un inmediato interés por el autor y su contexto que desembocó en un conjunto de monografías que se recopilaron tres años después en un nuevo volumen (Sansano y Valsalobre 2009). Tal efervescencia llevó a la formación de un equipo al que fueron concedidos dos proyectos de investigación del Ministerio de Economía y Competitividad sobre el poeta barcelonés: «Obra poética de Francesc Fontanella (1622-1682/83). Edición crítica» (2012-2015) y "Teatro y obra poética completa (II) de Francesc Fontanella. Edición crítica» (2016-2019). ${ }^{16}$ Es en el marco de estos proyectos, pues, que, desde el año 2012 se está llevando a cabo la edición crítica de la obra completa de Fontanella.

En un primer momento, se planteó la posibilidad de preparar una edición en formato libro, pero esta opción fue perdiendo fuerza, tanto por las dificultades que suponía encontrar una salida comercial a un corpus textual tan amplio y complejo (y presentado, además, en una edición erudita), como por la escasa repercusión que cabía esperar para una obra de estas características fuera del ámbito estrictamente académico.

Alternativamente a la publicación en papel, la vía digital aparecía como una opción muy atractiva, ya que sus costes eran asumibles dentro del limitado presupuesto del proyecto y se trataba de un formato que presentaba grandes ventajas: en primer lugar, permitía ofrecer una versión del texto, divulgativa y erudita a la vez, ya que sería el lector quien decidiría qué tipo de contenidos quería visualizar; en segundo lugar, una publicación digital, controlada por el mismo grupo, podría ser corregida y ampliada siempre que se considerara opor-

15. El Premi Lletra es una iniciativa de la Fundació Prudenci Bertrana y el proyecto Lletra de la Universitat Oberta de Catalunya, que se otorga en el marco de los Premis Literaris de Girona. Sobre la concesión de este galardón a Nise, véase <http://lletra.uoc.edu/ca/premi/premi-lletra-2013>.

16. Sobre el proyecto de edición crítica de la obra poética de Fontanella, véase Valsalobre (2015). 
tuno, una opción especialmente interesante cuando, como es el caso, se trabaja con textos antiguos, para los cuales pueden todavía aparecer nuevos testimonios y realizarse nuevos descubrimientos que ayuden a mejorar su comprensión.

A todo esto, había que añadir que, publicados en un entorno web, los textos pasaban a ser de dominio público, lo que significa que cualquier persona interesada podía acceder a ellos libremente siempre que lo deseara, y que el mismo grupo podía encargarse de contribuir a su difusión a través de la red. Además, la publicación en internet no estaba reñida con el papel, tal como pudimos comprobar con la publicación de la antología poética $O$ he de morir o he d'amar, preparada por Pep Valsalobre, Eulàlia Miralles y Albert Rossich, y editada por Empúries (Fontanella 2015).

Por último, y no menos importante, la adecuación que requería la web de Nise para poder presentar textos en formato de edición crítica permitiría, a la larga, no solo la publicación de los textos de Fontanella en este formato, sino los de los demás autores editados anteriormente o en el futuro por los miembros del grupo. Significaba, en definitiva, una oportunidad para ampliar las prestaciones de toda nuestra biblioteca digital.

Tomando en consideración todos estos aspectos, el grupo empezó a trabajar en la edición del corpus poético fontanellano en el año 2012. Desde el comienzo, se ha seguido un proceso bastante similar al que se habría seguido en el caso de querer publicar la edición en papel, una forma de trabajar que, en definitiva, permite obtener los textos en un formato que puede ser dirigido fácilmente a ambos medios. La metodología de trabajo ha consistido en asignar a cada investigador una serie de composiciones, sobre las que ha tenido que ejecutar tres operaciones: 1) la fijación del texto a partir del método neolachmaniano, es decir, del cotejo crítico de todos los testimonios que lo transmiten; 2) la regularización ortográfica del texto fijado, ${ }^{17}$ y 3 ) la elaboración de un aparato de notas que facilite la comprensión de los pasajes más complejos. Los resultados obtenidos se someten a discusión por parte de todos los miembros del grupo que participan en la edición y, posteriormente, los autores que firman este artículo se encargan de coordinar su publicación en la biblioteca digital de Nise.

En el momento de la redacción del presente trabajo se han publicado $175 \mathrm{com}-$ posiciones, lo que supone algo más de la mitad del corpus poético de Fontanella. También se ha empezado a preparar la edición de la obra dramática del autor, que consta de dos piezas mayores y tres piezas menores o fragmentarias. La previsión es que tanto la poesía como el teatro completo de Fontanella puedan estar disponibles en edición crítica electrónica dentro del web de Nise antes del año 2020.

17. Es importante precisar que la lengua catalana de época moderna, como las del resto de Europa, no presenta unos criterios ortográficos unificados. Por esta razón, se ha optado por seguir los criterios de regularización ortográfica definidos por Albert Rossich (Valsalobre y Rossich 2006: 25-36), que permiten dar a los textos unas grafías actualizadas, sin alterar la realización fonética de las palabras. 


\section{La biblioteca digital de Nise}

La publicación de los textos de Fontanella se inserta en la biblioteca digital de Nise, un espacio sobre el cual es importante realizar algunas aclaraciones previas.

La biblioteca, que todavía está en fase de desarrollo, se encuentra en la página web del grupo y se divide en dos partes: «Estudis» y "Autors». En la primera de ellas se pueden consultar las publicaciones académicas (artículos, libros, capítulos de libro, etc.) de los miembros del grupo de investigación, mientras que en la segunda se encuentran las páginas dedicadas a los distintos autores literarios, que aparecen por orden alfabético de apellido. ${ }^{18}$ Dentro de cada una de estas páginas, ordenados también de forma alfabética, se encuentran los textos de cada autor.

Esta segunda parte de la biblioteca, la que está dedicada a los autores y a sus obras, tiene una vocación inclusiva y sistemática, en el sentido que pretende dar acceso al usuario a cualquier obra literaria del período moderno en lengua catalana que pueda ser consultada en internet, independientemente de su formato y su editor. En la práctica, esto significa que en la biblioteca de Nise podemos encontrar textos de tres tipos: 1) ediciones en formato hipertextual creadas ad hoc por miembros de Nise (es el caso de la edición de Fontanella); 2) ediciones realizadas en formato papel por algún miembro de Nise y que, posteriormente, han sido publicadas en la web en formato de texto llano, pero que, a medio plazo, irán adquiriendo las mismas características que los textos del primer grupo (es el caso de Vicent Garcia y Agustí Eura); y 3) textos publicados en la red y a los cuales simplemente se da acceso a través de un enlace.

Una vez se accede a la página de un autor determinado, existen distintos mecanismos para informar al lector sobre el origen del texto que se dispone a leer. Por defecto, cuando se trata de una edición propia, el título de la obra o composición tiene la forma de un hipervínculo que, al ser clicado, dirige al usuario hacia la página donde se encuentra publicada. Al final de cada texto, se encuentra el apartado "Sobre aquesta edició", donde se indica si el texto en cuestión procede de una edición anterior en papel o, si como es el caso de los textos de Fontanella, ha sido publicado directamente en formato digital. En cambio, cuando se trata de una versión no producida por Nise pueden suceder dos cosas: que el texto solo haya sido editado por otro editor o que también haya sido editado por otro editor. En el primero de los casos, el título de la obra es un hipervínculo que conduce a otra página web, con lo cual se hace evidente que Nise no es responsable de la edición, y quizá no haría falta ofrecer más información al lector. No obstante, en casos como este, mediante el apartado «Font», debajo del título de la obra aparece la referencia de la edición a la cual se está por acceder.

18. Véase <http://www.nise.cat/ca-es/bibliotecadigital/autors.aspx>. 


\section{Figura 2.}

En el caso del poeta Pere Serafí, por ejemplo, dado que no disponemos de ninguna edición propia, proporcionamos al lector el acceso a dos reproducciones digitales de su obra poética ubicadas en la Biblioteca Virtual Miguel de Cervantes, cuya referencia aparece en el apartado «Font»

En cambio, cuando un mismo texto tiene otras versiones en internet, además de la de Nise, aparece, en la parte inferior del título de la obra, un enlace doble, con una opción que da acceso a la versión de Nise, y otra que da acceso a la otra versión.

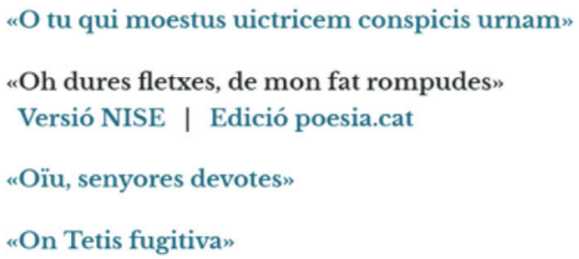

Figura 3.

En el caso del poema de Fontanella "Oh dures fletxes, de mon fat rompudes», ofrecemos la posibilidad de consultar la edición de Nise y la de la web poesía.cat.

\section{Criterios y objetivos de nuestra edición}

Dentro del apartado de la biblioteca dedicado a los autores, al escoger a Francesc Fontanella, accedemos a la página de presentación de la edición crítica de su obra poética. ${ }^{19}$ En esta página podemos leer una breve descripción de las características del proyecto y encontramos detallados distintos aspectos de la edición, entre los cuales, una relación de los testimonios cotejados, una lista con

19. Véase $<$ http://www.nise.cat/BibliotecaDigital/Autors/FrancescFontanella.aspx $>$. 
las ediciones impresas de textos de Fontanella, una bibliografía de las fuentes consultadas para la anotación de los textos y un documento donde se detallan los criterios seguidos para la regularización ortográfica de los mismos. ${ }^{20}$ También en esta página encontramos el acceso a la página dedicada a Fontanella, donde se hallan, por orden alfabético, los títulos de todos los textos publicados. Haciendo clic sobre los títulos llegamos, finalmente, a los textos.

La edición electrónica que hemos llevado a cabo se rige por tres criterios: adaptabilidad, hipertextualidad y compartibilidad.

\section{Adaptabilidad}

Entendemos que una edición digital puede ser al mismo tiempo erudita y divulgativa, y que nuestra obligación es proporcionar al lector los contenidos y las herramientas para que este pueda conformar sus propios itinerarios de lectura. Tal vez donde esto resulte más evidente sea en los aparatos críticos y en el apartado de información adicional que encontramos al final de cada texto.

La edición consta de tres tipos de aparatos: el de variantes, el de comentarios a las variantes y el de notas. El primero consigna las variantes textuales existentes entre el texto crítico y los testimonios, una información obtenida a partir del cotejo de todos los testimonios y de la aplicación de los criterios neolachmannianos de constitución del texto. El segundo aparato contiene menos información, ya que solo recurrimos a él cuando hace falta realizar alguna apreciación relativa a la lección editada o bien sobre algún aspecto destacado de alguno de los manuscritos (una corrección, una observación sobre el estado del manuscrito en un pasaje concreto del texto, etc.). El aparato de notas, por otra parte, sirve para proporcionar al lector algunos datos complementarios que le faciliten la comprensión y la interpretación del texto, y suele consistir en apreciaciones de tipo lingüístico, retórico o contextual.

Cuando existen contenidos para alguno de los tres aparatos mencionados, se pueden observar, en el margen derecho del texto, unos pequeños cuadrados de colores: rojo para el aparato de variantes, verde para los comentarios a las variantes y azul para las notas. La visualización de dichos símbolos puede ser activada o desactivada, conjuntamente o por separado, junto con la numeración de los versos, a gusto del lector, a través de un pequeño menú situado en la parte superior derecha del texto. De esta manera, si el usuario desea acceder a una versión del texto sin añadidos, no tendrá más que desactivar la visualización de los aparatos que no desee ver. $\mathrm{Si}$, por el contrario, desea que estén activos y quiere poder consultar en cada momento la información que le proporcionan, debe dejar la visualización activada (opción que se ofrece por defecto) y situar el cursor encima de los cuadrados. Esta acción conlleva la aparición de una ventana emergente, donde se muestra la información contenida dentro del aparato en cuestión.

20. Sobre esta cuestión, véase la nota 15 . 


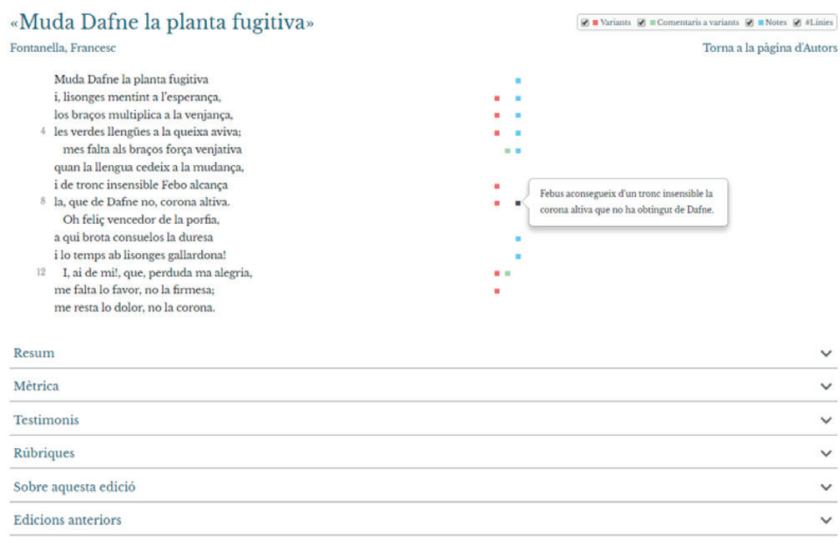

Figura 4.

Muestra de la edición de un soneto de Fontanella. En el margen derecho del texto, los cuadrados indican la presencia de contenidos de alguno de los tres aparatos críticos, cuya visualización se consigue al poner el cursor encima de ellos y puede ser desactivada desde el menú superior.

La adaptabilidad del texto a las necesidades del lector se hace patente, también, en los contenidos adicionales, un menú desplegable, que, como podemos observar en la figura anterior, encontramos al final de cada texto. Consta de un máximo de nueve apartados, seis de los cuales se muestran de forma permanente, porque siempre contienen información, y los tres restantes solo aparecen cuando tienen contenidos que mostrar. Son los siguientes: ${ }^{21}$

— «Resum», que presenta un breve resumen en prosa del contenido del texto.

- "Mètrica», donde se describen las formas métricas y estróficas del poema, así como el esquema de la rima.

- «Testimonis», que detalla todos los testimonios, ya sean manuscritos o impresos, cotejados para el establecimiento crítico del texto.

— «Rúbriques», que recoge, en transcripción paleográfica, las rúbricas de cada uno de los testimonios.

- «Sobre aquesta edició», que da cuenta de cómo se ha obtenido el texto en cuestión. Cuando no se indica que procede de una edición anterior, significa que el texto ha sido publicado directamente en la biblioteca digital de Nise.

21. Los apartados que pueden no aparecer son «Edicions anteriors», «Multimèdia» $\mathrm{y}$ «Referències bibliogràfiques». 
— «Edicions anteriors», que es un listado exhaustivo de las ediciones anteriores existentes.

- "Multimèdia», que da acceso a contenidos en formato audio o video relacionados con el texto en cuestión.

— «Referències bibliogràfiques», que es una relación detallada de las fuentes bibliográficas que se han ocupado anteriormente de aquel texto.

- «Descarrega aquest text», que permite la descarga de dos versiones distintas del texto: una que incluye el texto, el resumen y el aparato de notas y otra que, además de estos elementos, incluye también el aparato de variantes y el de comentarios a las variantes.

Los contenidos de estos apartados solamente se muestran si se hace clic sobre ellos, por lo que constituyen itinerarios de lectura posibles, pero no impuestos al lector. Dentro de estos apartados encontramos dos tipos de datos que, nuevamente, solo se hacen visibles por deseo expreso del lector: se trata de las referencias a los testimonios utilizados y a las fuentes bibliográficas consultadas. Los testimonios se identifican con unas siglas, y las fuentes bibliográficas y ediciones anteriores se citan por el sistema autor/fecha. Ambos tipos de citación tienen forma de hipervínculo, de manera que, si el usuario pasa el cursor por encima de ellos, se despliegan ventanas emergentes con la referencia completa del elemento citado.

\begin{tabular}{|c|c|}
\hline Resum & $\checkmark$ \\
\hline Mètrica & $\checkmark$ \\
\hline Testimonis & $\hat{\sim}$ \\
\hline Af. 133v; Be p. 6 ; B4 f. 99v; B5 f. 212; B5 f. 278v; B12 f. 81; C p. 262-263; CC; 12f. 156v-157; L.2 p. 71; L.4 p. 249-250; P p. 31; R p. 219; 22 & $\begin{array}{l}\text { Manuscrit } 261 \text { de la Biblioteca del Museu } \\
\text { Episcopal. Vic }\end{array}$ \\
\hline Rúbriques & $\checkmark$ \\
\hline Sobre aquesta edició & $\checkmark$ \\
\hline Edicions anteriors & $\checkmark$ \\
\hline
\end{tabular}

Figura 5.

La referencia completa del manuscrito 261 de la Biblioteca del Museu Episcopal de Vic se muestra a través de una ventana emergente al pasar el cursor por encima de su sigla (V2), que tiene formato de hipervínculo.

Por último, la edición que presentamos es adaptable, también, ya que ha sido diseñada en modo responsive, es decir, que se adecua a las características del dispositivo electrónico desde el cual se consulta. La única salvedad es que, en el caso de los dispositivos móviles, se recomienda la consulta en orientación horizontal, para una mejor distribución y visualización en pantalla de los distintos elementos que conforman el texto. 


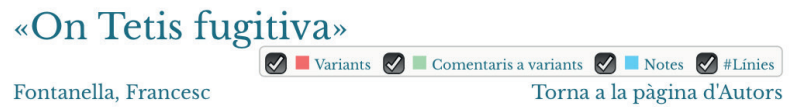

Fantasia no de músic

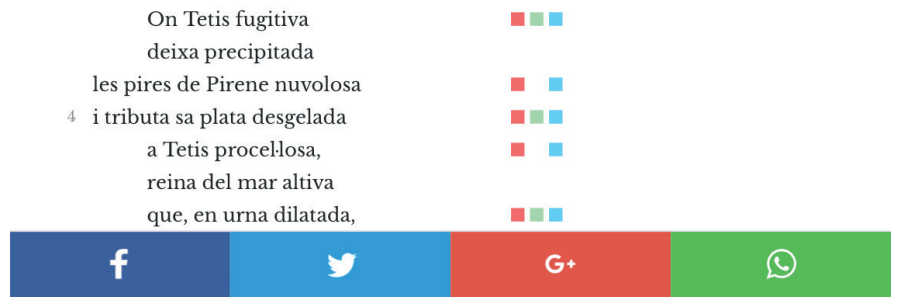

Figura 6.

Muestra del aspecto que presenta la edición consultada desde un teléfono móvil.

\section{Hipertextualidad}

Uno de los aspectos que consideramos más interesantes de un entorno digital es que ofrece la posibilidad de utilizar la gramática del hipertexto, es decir, el enlace de una parte concreta del texto con otras partes de otros textos o con otros tipos de contenidos. En el momento actual, esta característica de nuestra edición es, seguramente, la que se encuentra en un estado de desarrollo menos avanzado, pero aun así se han conseguido ya algunas prestaciones que consideramos importante mencionar aquí.

En un entorno web, los enlaces pueden apuntar hacia contenidos del mismo entorno o hacia contenidos que se encuentran fuera de este. Por el momento, la mayor parte de las rutas hipertextuales existentes en nuestra edición son del primer tipo. Este recurso resulta especialmente útil tanto para revelar las siglas de los testimonios y las fuentes bibliográficas - lo hemos visto en el apartado anterior-, como para poner de manifiesto la aparición de imágenes, metáforas, referencia, giros lingüísticos, etc., recurrentes en la obra del autor, así como cualquier tipo de relación existente entre diferentes textos. Es posible encontrar hipervínculos de esta clase tanto en el interior de los aparatos de notas, como en los apartados de contenidos adicionales de la parte inferior de la página. 


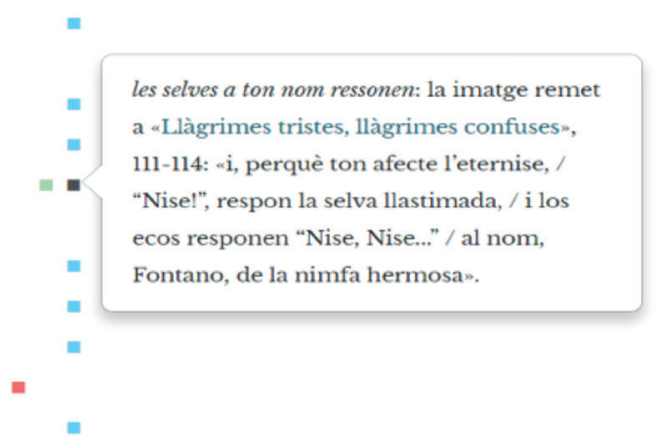

Figura 7.

Ejemplo de nota con hipervínculo a otro poema de Fontanella dentro del aparato de notas.

Además, como ya se ha dicho, la hipertextualidad puede también apuntar hacia fuera del entorno de la edición, y eso puede suceder tanto en el interior de los aparatos críticos, como en el interior de los contenidos adicionales. Dentro de estos últimos, resulta especialmente interesante poder proporcionar al lector contenidos multimedia, como el que se enlaza en el poema "De Fontano la queixa llastimosa», un vídeo en el que podemos ver al poeta y rapsoda Josep Pedrals recitando un fragmento del texto en el programa Àrtic, de Barcelona Televisió. ${ }^{22}$

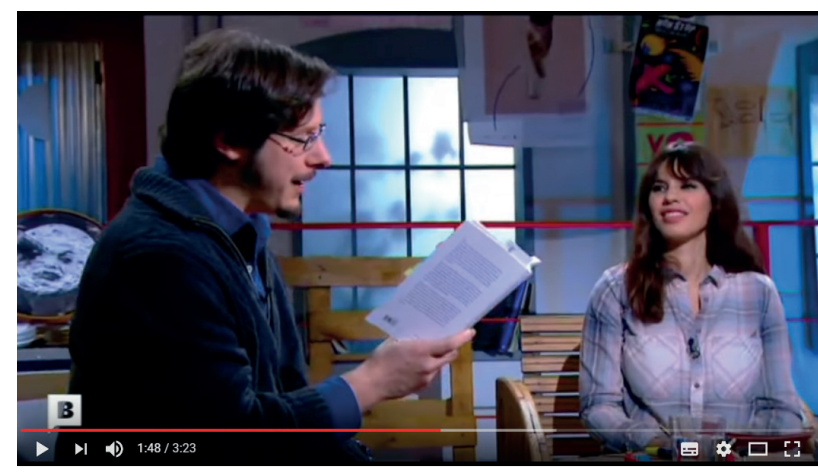

losep Pedrals parla de Francesc Fontanella

48 visualitzacions

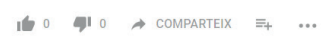

Figura 8.

Josep Pedrals recitando Fontanella en Barcelona Televisió.

22. Véase <https://www.youtube.com/watch?v=nFBKgnZ0jvQ >. 


\section{Compartibilidad}

Como ya se ha apuntado anteriormente, Fontanella, y los autores catalanes del periodo moderno en general, son, todavía hoy, autores poco conocidos dentro del mundo académico, pero sobre todo por parte del lector medio. Es por esta razón que la edición ha sido pensada para facilitar al máximo la circulación de los textos en las principales redes sociales. Así, en la versión web, al final de cada texto, encontramos los iconos de Facebook, Twitter y Whatsapp, que permiten compartir de manera rápida y fácil del texto en estas redes (Figura 9). En los dispositivos móviles estos iconos aparecen permanentemente en una franja horizontal en el borde inferior del marco de la imagen. (Figura 10).

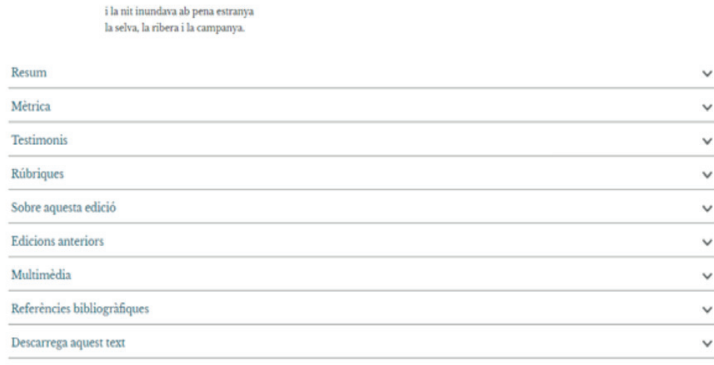

Figura 9.

Ubicación de los iconos de compartición en la página web.

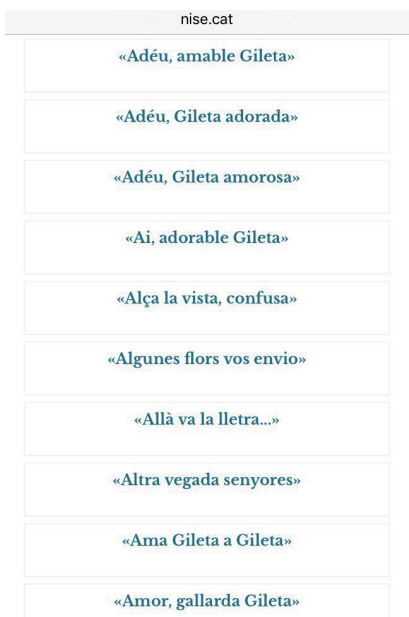

\section{f $y$ G. $\quad$ c}

Figura 10.

Ubicación de los iconos de compartición en la versión para dispositivos móviles.

Esta característica también resulta útil para Nise, que, paralelamente a la edición y publicación de los textos, lleva a cabo una difusión continuada de estos a través de sus perfiles en las redes sociales. Esta difusión será sostenida en el tiempo, con lo que se espera que pueda servir para dar a conocer la obra de Fontanella a los principales actores del sistema cultural catalán y a todo tipo de lectores. 


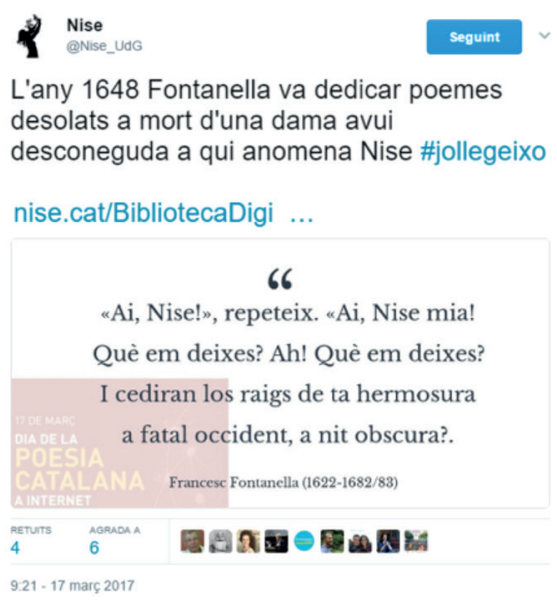

Figura 11.

Nise participó en la edición de 2017 del Dia de la poesia catalana a internet con tweets sobre los textos de Fontanella. ${ }^{23}$

\section{Conclusión: limitaciones y oportunidades}

Hasta aquí hemos descrito las principales posibilidades que ofrece, por ahora, nuestra edición, pero es bueno ser conscientes de que esta presenta también una serie de limitaciones. Algunas de ellas son inevitables mientras que otras van a ser corregidas en las siguientes fases del proyecto, durante los próximos años.

Seguramente, la principal limitación de nuestro proyecto es que se presenta en un formato editorial ad hoc, que fue diseñado por los autores del presente artículo e implementado por una empresa de servicios informáticos contratada específicamente con esta finalidad. ${ }^{24}$ Esta opción presentaba no pocos inconvenientes, siendo tal vez los más evidentes el hecho de depender de personal técnico ajeno al proyecto para su realización — con las dificultades y los costes económicos que esto supone- $\mathrm{y}$ el hecho de no estar trabajando en un formato de texto homologable en los estándares internacionales.

Entendemos que la opción de responsabilizarnos de todo el proceso hubiera sido óptima, pero el equipo de nuestro grupo de investigación es pequeño, y resultaba imposible que alguno de sus miembros pudiera dedicar su tiempo a la formación que hubiera sido necesaria para desarrollar la parte de diseño informático de la edición. A pesar de esta situación de partida, en el momento actual estamos trabajando para conseguir que el mismo aplicativo con el que se publica

23. Véase $<\mathrm{http}: / /$ lletra.uoc.edu/ca/projectes/dia-de-la-poesia-catalana-a-internet/detall $>$.

24. La empresa responsable del diseño del aplicativo es Disgrafic (<http://www.disgrafic.com/>). 
el texto sea capaz también de proporcionar una versión del mismo en formato xml, y que esta se pueda descargar desde el apartado «Descarrega aquest text», dentro de los contenidos adicionales.

Por otro lado, disponer de una estructura de publicación propia supone también ciertas ventajas. La más importante es que el resultado final es, cuanto a presentación e itinerarios de navegación, exactamente el que deseábamos que fuera, es decir, el que entendemos que proporciona una mayor libertad al lector y unas mayores posibilidades de acceder a aquellos detalles del texto por los que tenga más interés. El diseño propio es también una opción óptima desde el punto de vista de los investigadores que nos encargamos de publicar el texto, porque el aplicativo interno de la web a través del cual se realiza la publicación contiene exactamente los mismos apartados y en el mismo orden que los documentos de texto resultantes de nuestro trabajo de edición. Dicho de otra forma: para publicar el texto, prácticamente lo único que el investigador tiene que hacer es recortar cada fragmento (rúbrica, título, texto, aparato de variantes, comentarios a las variantes, aparato de notas, etc.) y pegarlo dentro del apartado correspondiente del aplicativo. Después, tan solo debe añadir algunas secuencias - muy mínimas- de código para introducir los hipervínculos dentro de los aparatos y los contenidos adicionales.

Una de las posibilidades que más a menudo se ha vinculado con la edición digital de textos es la de permitir al lector acceder a la versión digitalizada de los distintos testimonios, una posibilidad que resulta especialmente interesante cuando es posible coordinar su visualización con el del texto editado. Nuestra edición no ofrece esta opción por distintos motivos. El primero y principal es que, aunque algunas composiciones de Fontanella se encuentran únicamente en unos pocos testimonios, la mayor parte son transmitidas, al menos, por cinco o seis, llegando esta cifra, en numerosas ocasiones, a superar la decena. La visualización simultánea de todos estos testimonios, claro está, resultaría imposible.

Por otra parte, hay que considerar que la mayoría de los testimonios que transcriben la obra de Fontanella son manuscritos, y que solo una parte de ellos han podido ser debidamente digitalizados por las instituciones que los custodian. Se trata de un total de 33 testimonios, algunos de ellos de más de 300 folios, que se encuentran repartidos entre 15 centros documentales, así que los costes de su digitalización exhaustiva, la gestión de los derechos de reproducción digital y el esfuerzo de coordinación entre instituciones que toda esta operación supondría sobrepasaban, de mucho, las modestas posibilidades de nuestro proyecto. Además, habría que considerar que el interés de esta opción se circunscribe al público especializado y, en el caso de Fontanella, tratándose de un autor que todavía no es ni mucho menos conocido por el gran público, consideramos que resultaba más interesante apostar por aquellas funcionalidades de la edición que pudieran ayudar a acercar el autor a una mayor cantidad de lectores. A pesar de todo, nos parece altamente interesante poder llegar a incluir la digitalización de los testimonios en la edición, y por ello no descartamos llevarla a cabo en un futuro, si bien esta posi- 


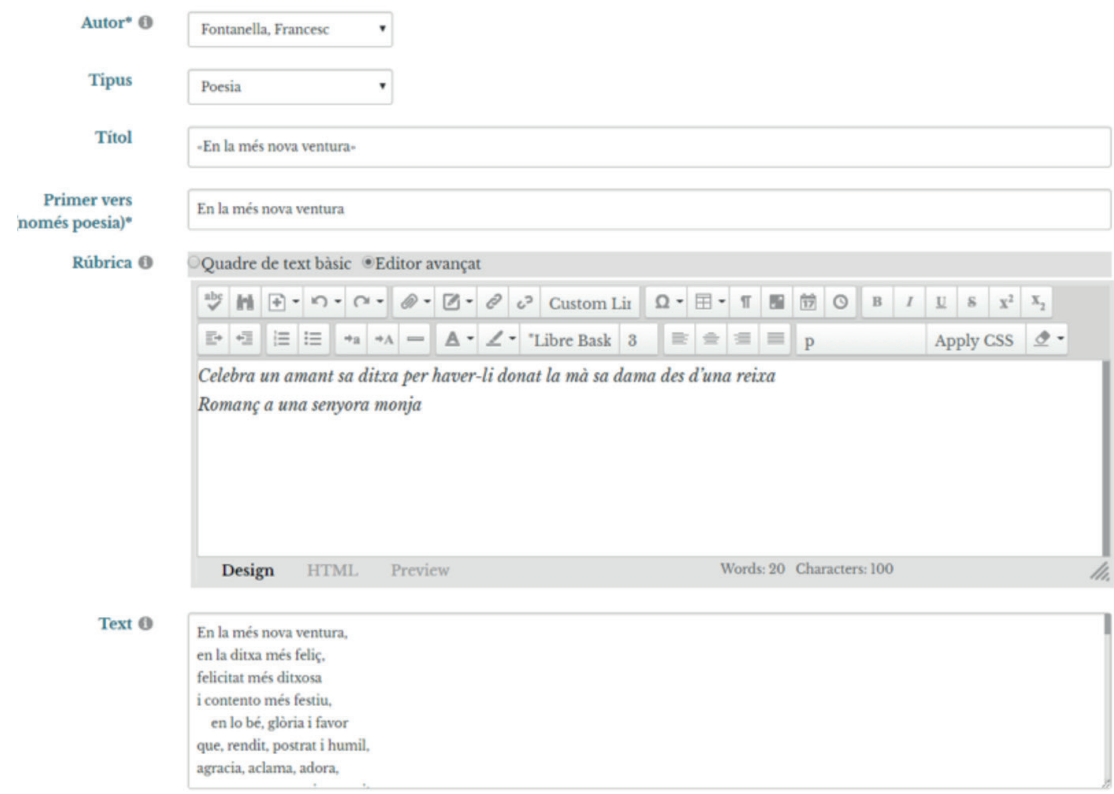

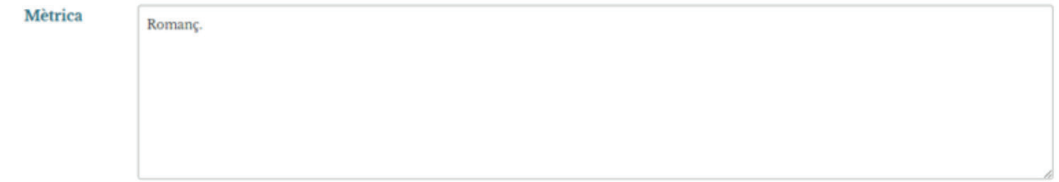

Testimonis 6 \$A\$ f. 175v-176v; \$B1\$ f. 11-12; \$B55 f. 269-270; \$B8\$ f. 88v-89; \$C\$ p. 181-183; \$CC\$; \$12\$ f. 104-104v; \$J\$ f. 131v-132; \$LA\$ p. 225-226; \$LAS' p. 288; \$P\$ p. 11; SR p. 288-289; \$V2\$ f. 129-130.

\$BIS: Celebra un amaci>n< $/ i>t$ la ditxa de haverli donat la ma sa dama p<i>er< $j>$ vna rexa. Romance

SB5\$: Romans a una señora monja

SB8S: Un amant agrait

SCS: Celebra un Amant sa ditxa per hauerly donat la ma sa Dama desde una rexa

\$12S: Celebra un amant sa ditxa per auerli donat la ma sa dama des $<$ jode $/ i>$ una reixa

SJS: A un favor de una ma. R[oman]s

Edicions 6 anteriors

\$Miró (1995: 1, 266-268)S; SValsalobre, Miralles \& Rossich (2015: 159-161)\$.

Figura 12.

Vista del aplicativo a través del cual se lleva a cabo la publicación de los textos. Dentro de "Testimonis», «Rúbriques» y «Edicions anteriors» podemos observar los símbolos \$, que sirven para programar las ventanas emergentes. La información que se presenta en estas ha sido introducida previamente en una base de datos aparte, dentro del mismo sitio web. 
bilidad quedará supeditada al hecho de poder disponer de los recursos necesarios para llevar a cabo un proyecto específicamente diseñado para este fin.

En cambio, otra opción que nuestra edición por ahora no incluye pero que sí esperamos incorporar en un futuro próximo son los motores de búsqueda, que permitan encontrar, dentro de un corpus textual concreto (en este caso, el de Fontanella) palabras, palabras-rima, formas métricas, y otros cruces de datos.

También tenemos previsto ampliar la profundidad y la riqueza del hipertexto. En este sentido, además de completar las interacciones internas entre textos, las cuales, sin duda, aumentarán a medida que vayamos ampliando el corpus de composiciones publicadas y avanzando en el estudio de la vida y la obra del autor, intentaremos, también, expandir los límites del texto ofreciendo contenidos en formato audiovisual. Una opción que nos parece especialmente interesante es incluir las audiolecturas de los textos, para aquellos lectores que prefieran escuchar el texto además o en vez de leerlo.

En otro orden de cosas, también será necesario introducir algunos cambios en la organización del acceso a los textos de Fontanella. Actualmente, las composiciones poéticas aparecen en orden alfabético, lo que facilita su localización siempre y cuando el lector conozca el primer verso de la composición. Pero esta ordenación, aunque pueda resultar práctica para un determinado tipo de lector, presenta un problema muy evidente, y es que impide visualizar el orden de los textos en los manuscritos. Este orden es importante, porque muchas de las composiciones de Fontanella conforman unidades de sentido (Valsalobre 2015b), de manera que resulta interesante poder leerlas en el orden correcto. En este sentido, presentar todas las ordenaciones de todos los manuscritos sería una opción óptima, pero muy costosa y no del todo eficaz, ya que, aunque los testimonios que transmiten la obra del autor son muchos, ninguno la transcribe completa, y solo uno, el manuscrito 68 de la Biblioteca Lambert Mata de Ripoll, ${ }^{25}$ parece presentarla en el orden deseado por el autor. Por eso, una de las ordenaciones que ofrecerá en el futuro la edición de Fontanella será la del acceso a los textos en el orden dado por este manuscrito, así como la posibilidad de acceder a las composiciones a partir del título de los distintos ciclos literarios que componen.

Todavía en relación al acceso a los textos, tal como se ha dicho más arriba, actualmente estamos realizando también la edición de la obra dramática de Fontanella, y el objetivo es crear, para esta parte del corpus, una entrada independiente, que, en la página de inicio de la edición, se ofrecerá junto con el acceso a la obra poética. Además, los textos teatrales requerirán algunos añadidos en el diseño de la visualización del texto editado, aunque mínimos y relacionados con la navegación. Nuestro propósito, en este sentido, es que, al inicio de la obra, en formato hipertextual, el lector encuentre un esquema de las divisiones de esta en actos y escenas, de manera que pueda acceder desde allí a la parte de la obra que desee.

25. Sobre la importancia de este manuscrito, véanse Miralles (2015) y Rossich y Miralles (2014). 
Para terminar, pensamos que una de las mayores oportunidades que nos ofrece el medio digital es la de realizar mejoras, correcciones y añadidos a lo largo del tiempo. En este sentido, esperamos ir ampliando el contenido de la edición con los resultados que, sin duda, la investigación sobre Fontanella va a ofrecer en los dos ańos que quedan del proyecto. Tanto en la corrección de errores, como en la ampliación de contenidos, esperamos, también, poder contar con una ayuda de los mismos usuarios de la edición. Aunque esta opción no está disponible actualmente y el mecanismo está todavía por definir, nuestro objetivo es que en esta última fase del proyecto sea posible introducir algún tipo de funcionalidad que permita al lector realizar sus propuestas, comentarios y observaciones, que luego valoraremos y, de considerarlos oportunos, añadiremos a la edición. 


\section{Bibliografía}

Eura, Agustí, Obra poètica i altres textos. Edició crítica, ed. Pep Valsalobre, Barcelona, Fundació Pere Coromines, 2002.

Fontanella, Francesc, $O$ he de morir o he d'amar, eds. Valsalobre, Pep; Miralles, Albert; Rossich, Albert, Barcelona, Empúries, 2015.

Garcia, Vicent, Francesc Vicent Garcia, Antologia poètica, Albert Rossich, Santes Creus-Barcelona, Fundació Roger de Belfort, 1985.

MarTí, Sadurní, «Cançoners DB: A new research tool for the study of medieval Catalan songbooks», Digital philology: A Journal of Medieval Cultures, vol. 3, n. ${ }^{\circ} 1$ (2014), 24-42.

Martínez-Gil, Víctor (coord.), Cerdì, Jordi; Gavagnin, Gabriella; Martí, Sadurní; Miralles, Eulàlia; Pujol, Josep; Valsalobre, Pep, Models i criteris de l'edició de textos, Barcelona, Editorial UOC, 2013.

Miralles, Eulàlia, «Algunes reflexions sobre la disposició textual d'un cançoner barroc (BLM, ms. 68). Per a una lectora: un llibre-ofrena i un testament literari», Zeitschrift für Katalanistik, 28 (2015), 187-230.

Miralles, Eulàlia (ed.), Del Cinccents al Setcents. Tres-cents anys de literatura catalana, Bellcaire d'Empordà, Vitel.la, 2010.

Puig, Pau, Obra catalana, ed. Maria Rosa Serra, Barcelona, Barcino, 2012.

Ramis, Joan, Arminda, eds. Vicent de Melchor y Pep Valsalobre, Bellcaire d'Empordà, Edicions Vitel.la, 2006.

Rossich, Albert, Francesc Vicent Garcia: assaig d'edició crítica [tesis doctoral], Bellaterra, Universitat Autònoma de Barcelona, 1985.

—, «És vàlid avui el concepte de Decadència de la cultura catalana a l'època moderna? Es pot identificar decadència amb castellanització?», Manuscrits, 15 (1997), 127-134.

-, (coord.), Panorama critic de la literatura catalana. III. Edat Moderna, Barcelona, Vicenç Vives, 2011.

Rossich, Albert; Miralles, Eulàlia, «Un pròleg desconegut de Francesc Fontanella», Els Marges, 102 (2014), 90-102.

Rossich, Albert; VAlsalobre, Pep, Literatura i cultura catalanes (segles XVI-XVIII), Barcelona, Editorial UOC, 2007.

-, Literatura catalana moderna (siglos XVI-XVIII), Madrid, Editorial Síntesis, 2011.

Sansano, Gabriel; Valsalobre, Pep (ed.), Fontanellana: estudis sobre l'època $i$ l'obra de Francesc Fontanella (1622-1683/85), Girona, Documenta Universitaria, 2009.

Valsalobre, Pep, «Per a una edició crítica de la poesia de Francesc Fontanella», Els Marges, 105 (2015a), 84-107.

—, «Sobre la transmissió manuscrita de l'obra poètica de Francesc Fontanella: els cançoners principals i l'ordenació dels textos», Zeitschrift für Katalanistik, 28 (2015b), 167-186. 
Valsalobre, Pep; Rossich, Albert, Poesia catalana del barroc. Antologia. Bellcaire d'Empordà, Edicions Vitel.la, 2006.

Valsalobre, Pep; Sansano, Gabriel (eds.), Francesc Fontanella: una obra, una vida, un temps, Bellcaire d'Empordà, Edicions Vitel.la, 2006.

Veny-Mesquida, Joan Ramon, Criticar el text. Per a una metodología de l'aparat crític d'autor, Lleida, Aula Màrius Torres/Pagès Editors, 2015.

Zaragoza, Verònica, "En vers vull desafiar...". La poesia femenina a l'àmbit català (segles XVI-XVIII). Edició crítica [tesis doctoral], Girona, Universitat de Girona, 2016.

Webgrafia [última consulta de estas webs 15/07/17]

$<$ http://damaboba.unibo.it>

$<$ http://letra.uoc.edu/ca/premi/premi-lletra-2013>

$<$ http://lletra.uoc.edu/ca/projectes/dia-de-la-poesia-catalana-a-internet/detall >

$<$ http://tc12.uv.es>

$<$ http://whitmanarchive.org $>$

$<$ http://www.bovary.fr>

$<$ http://www.catedramariustorres.udl.cat>

$<$ http://www.disgrafic.com/>

$<$ http://www.narpan.net>

$<$ http://www.narpan.net/bibliotecadigital/biblio-digital.html>

$<$ http://www.nise.cat/ca-es/ca-es/bdpoesiaencançoners/sobreaquestabasededades. aspx>

<http://www.nise.cat/ca-es/bibliotecadigital/autors.aspx>

$<$ http://www.nise.cat/BibliotecaDigital/Autors/FrancescFontanella.aspx>

$<$ http://www.rialc.unina.it>

<https://www.youtube.com/watch?v=nFBKgnZ0jvQ> 